\title{
Flavor Decomposition of Nucleon Spin Structure: A Proposed Experiment at Jefferson Lab Hall C
}

\author{
Xiaodong Jiang*, Donal B. Day ${ }^{\dagger}$ and Mark K. Jones** \\ *Department of Physics and Astronomy, Rutgers University, Piscataway, New Jersey. \\ ${ }^{\dagger}$ Department of Physics, University of Virginia, Charlottesville, Virginia. \\ ${ }^{* *}$ Thomas Jefferson National Accelerator Facility, Newport News, Virginia.
}

\begin{abstract}
We propose to measure the semi-inclusive double-spin asymmetries in deep-inelastic $\vec{p}\left(\vec{e}, e^{\prime} h\right) X$ and $\vec{d}\left(\vec{e}, e^{\prime} h\right) X$ reactions $\left(h=\pi^{+}, \pi^{-}, K^{+}\right.$and $\left.K^{-}\right)$on longitudinally polarized $\mathrm{NH}_{3}$ and $\mathrm{LiD}$ targets. The high statistic data will allow a leading order five-flavor decomposition $(\Delta u$, $\Delta d, \Delta \bar{u}, \Delta \bar{d}, \Delta s=\Delta \bar{s}$ ) of the nucleon spin structure in the region of $x=0.12 \sim 0.43$ at a $Q^{2}$ of $1.21 \sim 3.22 \mathrm{GeV}^{2}$. The combined spin dependent $\pi^{+}+\pi^{-}$yield ratios will be measured, since the acceptance and the hadron efficiencies of the detectors can be accurately determined. Independent of fragmentation functions and parton distributions, the z-dependence $(z=0.38 \sim 0.71)$ of the combined ratios provides a build-in test of factorization at the moderate $Q^{2}$. We estimate statistical uncertainties associated with such measurements.
\end{abstract}

\section{INTRODUCTION}

At the Thomas Jefferson National Accelerator Facility, the combination of a CW polarized electron beam and the use of polarized targets presents many new physics opportunities, especially in the measurements of spin observables of deep inelastic semiinclusive scattering (SIDIS). If factorization between quark scattering and quark fragmentation can be clearly demonstrated, SIDIS can provide accesses to quark polarization distributions. In the current fragmentation regime, the leading hadron from the fragmentation is strongly correlated with the struck quark, and the virtual photon asymmetry $A_{1}^{h}$ is related to quark polarization at the leading order as:

$$
A_{1}^{h}\left(x, Q^{2}, z\right)=\frac{\sum_{f} e_{f}^{2} \Delta q_{f}\left(x, Q^{2}\right) \cdot D_{f}^{h}\left(z, Q^{2}\right)}{\sum_{f} e_{f}^{2} q_{f}\left(x, Q^{2}\right) \cdot D_{f}^{h}\left(z, Q^{2}\right)} .
$$

Once factorization can be demonstrated at a reasonable level, flavor decomposition of nucleon spin structure can be achieved based on data sets of double-spin asymmetries [1]. In this proposed experiment, we will use asymmetries of $A_{1 p}^{\pi^{ \pm}}, A_{1 p}^{K^{ \pm}}, A_{1 d}^{\pi_{1}^{ \pm}}, A_{1 d}^{K^{ \pm}}$ and the better-known inclusive asymmetries $A_{1 p}$ and $A_{1 d}$.

To quantitatively demonstrate factorization in SIDIS, it is crucial to perform precision measurements on observables that are sensitive to the violation of factorization. A schematic strategy of factorization tests was suggested [2] which requires no prior knowledge of fragmentation functions nor parton distributions. The major experimental challenge in this strategy is to observe the $z$-dependence of the combined $\pi^{+}+\pi^{-}$ helicity asymmetries. 


\section{THE PROPOSED MEASUREMENTS}

We propose to measure the $\vec{p}\left(\vec{e}, e^{\prime} h\right) X$ and $\vec{d}\left(\vec{e}, e^{\prime} h\right) X\left(h=\pi^{+}, \pi^{-}, K^{+}\right.$and $\left.K^{-}\right)$spindependent cross sections with longitudinally polarized $\mathrm{NH}_{3}$ and LiD targets in Jefferson Lab Hall $\mathrm{C}$ with a $6 \mathrm{GeV}$ polarized electron beam [3]. The existing HMS spectrometer will be located at $10.8^{\circ}$ as the hadron arm detector. Three HMS momentum settings $(2.00,2.62$ and $3.22 \mathrm{GeV} / \mathrm{c})$ will be taken for both positive and negative polarities. A large calorimeter array, with a solid angle of $200 \mathrm{msr}$, will be centered at $30^{\circ}$ as the electron arm in conjunction with a threshold gas Cherenkov detector. A polarized proton luminosity of $8.5 \times 10^{34} \mathrm{~cm}^{-2} \mathrm{~s}^{-1}$ with $80 \%$ polarization can be achieved with the UVa polarized $\mathrm{NH}_{3}$ target. For a total of 24 days of beam time, the expected statistical errors on $A_{1 p}^{\pi}$ and $A_{1 d}^{\pi}$ are shown in Fig. 1 , and the expected errors on $A_{1 p}^{\pi^{+}+\pi^{-}}$are shown in Fig. 2. The expected accuracies on the spin-flavor decomposition are shown in Fig 3.

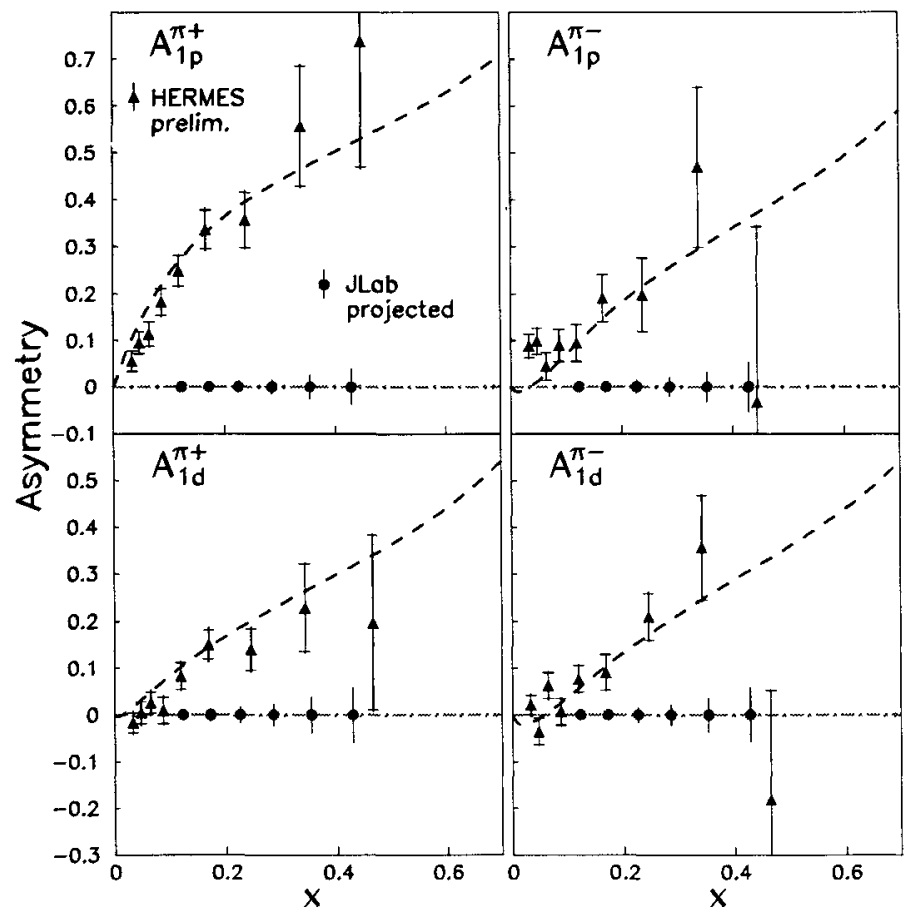

FIGURE 1. The expected statistical accuracy of $A_{1 p}^{\pi^{+}}, A_{1 p}^{\pi^{-}}, A_{1 d}^{\pi^{+}}$and $A_{1 d}^{\pi^{-}}$as functions of $x$. The HERMES preliminary results [1] are also shown. The dashed lines are the expected asymmetries for $\left\langle Q^{2}\right\rangle=2.5 \mathrm{GeV}^{2}$ and $\langle z\rangle=0.5$ using the CTEQ5 and the AAC quark distributions. 


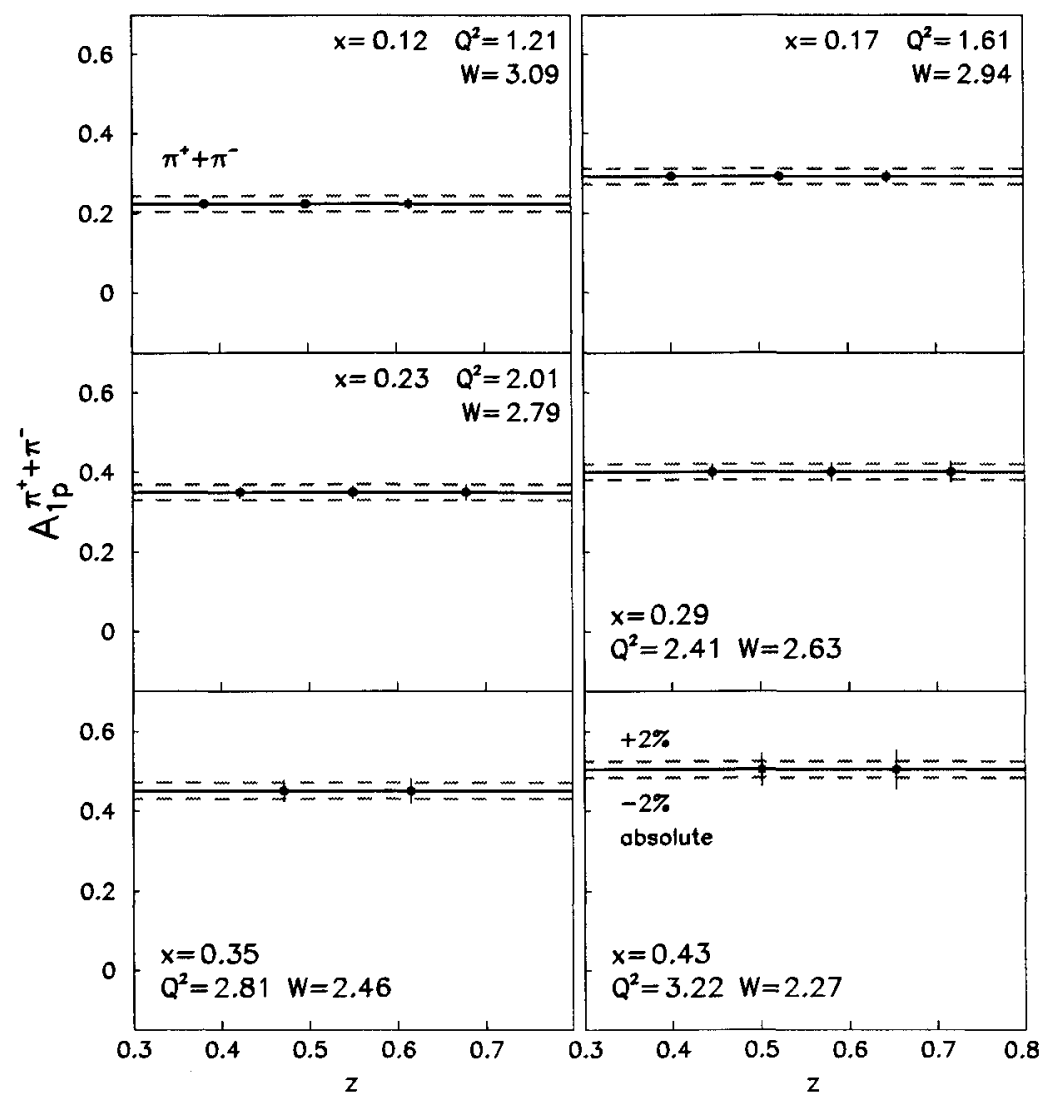

FIGURE 2. The expected statistical accuracy of spin-dependent yield ratio $A_{1 p}^{\pi^{+}+\pi^{-}}$as functions of $z$ are plotted for each $x$-bins. The solid line indicates the expected value from the factorization assumption, CETEQ5 and AAC parameterization. The dashed lines indicate an absolute variation of $\pm 2 \%$.

\section{ACKNOWLEDGMENTS}

The authors thank R. Ent, J.-P. Chen, E.Christova, E. Leader for many discussions. This work is supported by the US Department of Energy and the National Science Foundation.

\section{REFERENCES}

1. HERMES collaboration, hep-ex/0307064; A. Miller, plenary talk at the SPIN2002 Conference, Sept 9-14, Long Island, New York, USA; B. Beckmann hep-ex/0210049.

2. E. Christova and E. Leader, Phys. Lett. B468, 299 (1999) and Nucl. Phys. B607, 369 (2001).

3. "Flavor Decomposition of Nucleon Spin Structure", Jefferson Lab proposal PR-03-114, D. B. Day, X. Jiang, M. K. Jones co-spokespersons. 


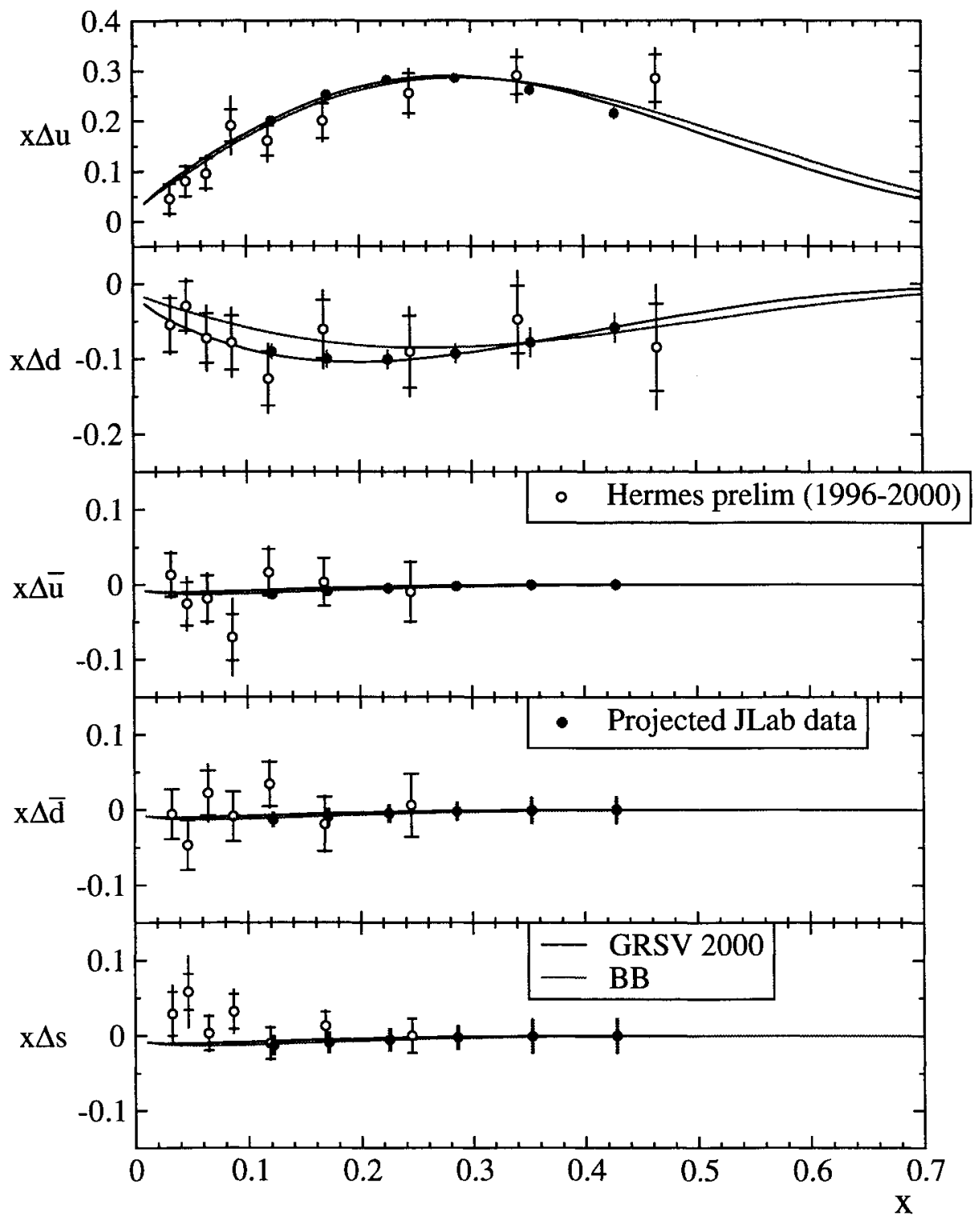

FIGURE 3. The expected statistical accuracy of 5-flavor decomposition compared with the preliminary HERMES data (1996-2000). The error bars on the projected JLab data are statistical only while the HERMES data includes both statistical (inner bars) and the systematic errors. 